\title{
Theoretical and experimental control strategies assessment of a Sliding Vane Oil Pump
}

\author{
Fabio Fatigati ${ }^{1}$, Marco Di Bartolomeo ${ }^{1}$, Giuseppe Lo Biundo ${ }^{2}$, Francesco Pallante ${ }^{2}$, \\ Roberto Cipollone ${ }^{1}$ \\ ${ }^{1}$ Department of Industrial and Information Engineering and Economics, University of L'Aquila, \\ Piazzale Ernesto Pontieri, Monteluco di Roio, 67100, L'Aquila, Italy \\ ${ }^{2}$ O.M.P. Officine Mazzocco Pagnoni S.r.L., Via Marzabotto, 71/73, 40050 Argelato (BO), Italy
}

\begin{abstract}
To date, Sliding Vane Pump (SVP) technology is one of the most attractive solution in different technical applications thanks to its reliability and compactness and capability to keep a high efficiency even when it is working far from rated condition. In particular, this feature makes the SVP suitable to be employed for the oil circulation (SVOP) in Internal Combustion Engine (ICE) which is characterized by a wide oil flow rates variation, delivered pressure and temperature variation which causes operating conditions of the pump far from the design point. Flow delivered changes in these machines are produced by varying the eccentricity - for a mechanical connection with the engine - or by varying the speed of revolution. The mild hybridization of the powertrains calls for a strong development of electrically assisted engine auxiliaries which undoubtedly makes the flow variations easier to be done, but the presence of an electric motor requires some technological choices not fully assessed, a cost increase and a reliability decrease. The paper presents a mathematical model of a SVOP for oil circulation in ICE, suitably validated by a wide experimental activity. The model integrates a mono and zero-dimensional fluid-dynamic analysis and allows to represent the intimate behaviour of the machine. Moreover, it was employed as virtual platform to discuss pros and cons of different flow rate variation strategies and their effect on the efficiency of the SVOP.
\end{abstract}

\section{Introduction}

New regulations in homologation cycles related to $\mathrm{CO}_{2}$ emissions and to other pollutants fixed by the EURO directives have led the scientific research in finding new solutions to increase efficiency and fuel economy in internal combustion engines (ICE). In this context, beside thermodynamic improvements, also parasitic losses referred to the engine auxiliaries can be targeted in order to improve the overall performances of the engine in terms of $\mathrm{CO}_{2}$ emitted, the driver of the technological evolution of ICE. In particular lubrication circuit and, more specifically, the engine oil pump shows a good room of improvement. Being the pump of a volumetric type, the flow rate delivered is almost linearly proportional to the pump speed which is mechanically connect to the engine shaft. This unavoidably requires systems which limit the flow rate when the oil quantity becomes excessive. In fact, a surplus of flowrate occurs at high speed and a pressure relief valve (PRV) is necessary in order to maintain oil pressure at a suitable value: this certainly represents a source of power lost. This issue could be addressed through an electric actuation of the pump [1] which would lead to the downsizing of the pump adding a further degree of freedom. In [2] Yamamoto et al. developed a unit comprising a drive, and electric motor and an internal gear pump optimizing total length of the unit and the clearances over the whole temperature operating range of the 
oil reaching a maximum $50 \%$ global efficiency. However electric actuations are not easy to be implemented and could not satisfy reliability standards of internal combustion engines. Most of the available scientific studies on conventional systems addressed this problem trying to optimize the geometry features of gerotor oil pumps. In [3] the authors considered a series of modifications related to the geometry of the discharge chamber, relief cut and rotor clearances optimization in a gerotor pump achieving a $18 \%$ power reduction which lead to a decrease in fuel consumption of around $1 \%$. In [4] similar procedures were applied in order to adapt a similar passenger car gerotor oil pump to a low viscosity oil, operating at higher temperature. A $15 \%$ power consumption decrease together with a $15 \%$ higher flowrate have been achieved. In [5], an existing oil pump was modified acting on the rotor thickness and clearances resulting in a $7 \%$ higher flowrate at idling speed and a $2 \%$ power reduction together with an improvement of the oil pressure and a quicker priming which is particularly beneficial in cold start conditions, Similar results have been achieved in [6] and [7] in engines of different nature showing an $18 \%$ power reduction with respect to the OEM case and an $11 \%$ oil pressure increase respectively. In [8], a CFD study on a high-performance motorbike oil pump is presented in order to study cavitation phenomena at high speed regime. Results agree with experimental results showing that $3 \mathrm{D}$ numerical models can be efficiently used during the design phase of oil pumps in anomalous conditions. A new design of a gerotor pump with variable discharge is presented in [9]. The idea is to conceive solutions in which the delivering of oil flowrate is not only related to the speed of rotation but also of some geometrical aspects which can be varied in different ways through mechanical or electric actuations, [10]. An innovative tooth profile determined 34\% friction reduction, controlling the pressure delivered over the whole range of operating conditions with good dynamic response. The new design has been tested on the Los Angeles driving cycle \#4 reducing fuel consumption by $1.2 \%$.

More recently Sliding Vanes Oil Pumps have been studied. This kind of positive displacement pumps generally show higher total efficiencies but the geometrical complexity has delayed their wide commercial development. The study presented in [11] combined SVOP with a variable displacement mechanics which reduced power consumption at high speed by $40 \%$. Arata et al. [12] presented a mechanically actuated variable displacement whose result was to have higher flowrate at low speed with a reduced power consumption at high loads. In [13] a variable displacement SVOP has been tested: it has been operated in two different modes, with a fixed and a variable eccentricity. Results showed that a $2.5 \%$ improvement in brake specific fuel consumption was obtained but at the expense of higher NOx emissions due to higher oil temperature. Modelling aspects are discussed in [14]. A 1D approach using GT-Suite ${ }^{\mathrm{TM}}$ platform was used. The model was able to reproduce friction phenomena and dynamic behaviour of the oil related to pressure fluctuations in addition to oil flowrate increase. Experimental data have been used to validate the model and define the major sources of friction. In [15] a mathematical model of a variable displacement SVOP has been built showing flowrate deviation from experimental data ranging from $1.5 \%$ to $7 \%$ in the whole operating range of the engine. Results confirmed also literature findings showing lower power consumption with respect to conventional technologies at high speed regimes. Rundo et al. went deep into the modelling aspect of this kind of pumps. In [16] a couple 3dod model is presented. CFD simulations are used in order to define some of the input parameter of the 0 -d model which is able to reproduce dynamic data related to pressure fluctuations. The model has been further developed in [17] in order to take into account also the aeration conditions of the oil. Issues related to pressure measurements inside the chambers of the pump are discussed in [18]. In particular it shows some relevant aspect related to the correct positioning of pressure transducers in order to correctly measure the evolution of the pressure inside the vanes without experiencing peaks of pressure which are not related to the real phenomena happening in the closed volumes. 
Thus, Sliding Vane Oil Pump (SVOP) allows to achieve a high flexibility rate in terms of flow rate variation obtained by varying speed of revolution and eccentricity: devices which improves the priming is also achievable as well as the limitation of internal overpressures due to the oil compressibility which is quite variable as a function of operating temperature. An important upgrade of this type of oil pumps are related to the possibility to easily modify its eccentricity relating SVOP operating condition. Moreover, a future issue is represented by the electrification of the pump which will offer a relevant degree of freedom for the pump design.

In order to support future developments and assist them with a virtual software platform able to represent the intimate fluid-dynamic behaviour of SVOP and the effects of control strategies, in this paper a numerical model was developed and validated thanks to a wide set of experimental data. The model is fully physically based and mixes a 1D approach during filling and emptying processes through ports and a lumped parameter model to reproduce properties inside closed vanes.

\section{SVOP mathematical model}

The numerical model of the SVOP was developed in GT-Suite ${ }^{\mathrm{TM}}$ environment integrating a zero-dimensional (0D) and a mono-dimensional analysis (1D). In particular, the 1D analysis was used to represent the fluid-dynamic phenomena taking place in correspondence of intake and exhaust pipes. Indeed, due the intrinsic transient nature of such phenomena, the intake and exhaust pipes were discretized (Fig.1) and for each sub-volume the Navier-Stokes equations were solved. 1D analysis was applied to reproduce the filling and emptying of the pump chambers, one of the most important process really important when oil properties are changed due to oil temperature. Once the vanes were filled (partially or completely), each chamber was treated as a lumped capacity. According to the oil compressibility (significantly temperature dependent), the pressure inside the closed vanes was calculated using basic thermodynamics accounting for the reduced volume variation during rotation. Additional refined modelling was introduced in order to limit overpressures. Thanks to this approach, the pressure inside the vane can be evaluated (and also the temperature) allowing the evaluation of the indicated power (1) where $\mathrm{p}_{\mathrm{i}}$ and $\mathrm{V}_{\mathrm{i}}$ are respectively the pressure and volume (which vary during rotation) of the considered i-chamber.

$$
P_{\text {ind }}=\frac{\oint \sum_{i=1}^{N v} p_{i} d V_{i}}{t_{\text {cycle }}}
$$

The indicated power $\mathrm{P}_{\text {ind }}$ represents the mechanical power exchanged between the machine (rotating vanes) and the fluid. It is generally higher than the hydraulic power $\mathrm{P}_{\text {hydr }}$ given by the product between the volume flow rate and the pressure rise (2).

$$
P_{\text {hydr }}=Q \Delta p
$$



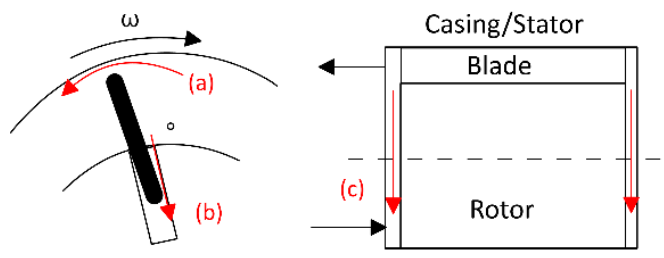

(a) Blade tip-Stator Leakage

(b) Blade side-Rotor Leakage

(c) Casing-rotor Leakage

(d) Recirculated flow at seal arc (tangency)

Volumetric losses assessment

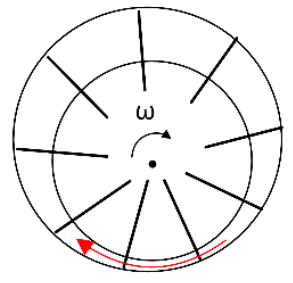

(d)

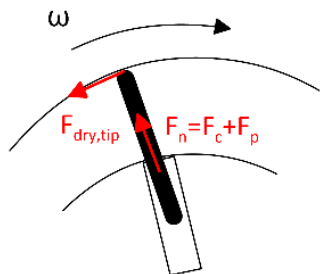

Mechancial losses assessment

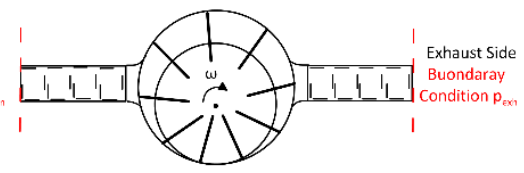

$\mathrm{F}_{\mathrm{dry}}$ - Friction Force at tip blade

$\mathrm{F}_{\mathrm{n}}$ - Normal Force

$\mathrm{F}_{\mathrm{c}}$ - Centrifugal Force

$F_{p}$ - Force exerted by the fluid under the blade

Fig. 1. SVOP numerical model : Volumetric and Mechanical losses assessment

As it is known, $\mathrm{P}_{\text {ind }}$ is greater than $\mathrm{P}_{\text {hydr }}$ due to the fact that pressure losses occur during the intake phase and discharge and to other phenomena which influence the pressure inside the vane (leakages which produces volumetric efficiency deficits, fluid dynamic losses inside vanes, heat transfer, etc...). The $0 \mathrm{D}$ analysis allows to evaluate the volumetric losses taking place in the leakage paths summarized in Fig.1: (a) Between blade tip and stator inner surface; (b) Between blade side and rotor slot; (c) Between rotor face and casing. If the eccentricity was reduced with respect the rated one, another leakages path takes place at the sealing arc (d) which can be interpreted as a portion of the fluid which is compressed and re-expanded periodically. All the leakages flows were calculated through a lumped approach, (a) and (b) were treaded using the Poiseuille/Couette relation [19], (c) was taken into account through an equivalent orifice model; (d) was evaluated considering the volume of the chamber in correspondence of the seal arc (tangency). Concerning the clearance gaps $\delta_{(\mathrm{a})}\left(\delta_{\text {tip }}\right)$, it has been identified through an experimental activity according the approach followed in [20] being really difficult to measure it during rotation. $\delta_{(\mathrm{b})}$ and $\delta_{(\mathrm{c})}$ on the contrary were geometrically identified. The knowledge of volumetric losses allows to assess the volumetric efficiency $\eta_{\mathrm{vol}}$ of the machine, expressed according (3):

$$
\eta_{v o l}=\frac{Q}{V_{d i s p} \omega}
$$

being $\mathrm{V}_{\text {disp }}$ geometrically fixed and $\mathrm{Q}$ measured by a flowmeter. The model allows to evaluate also the mechanical losses $\mathrm{P}_{\text {mech,lost }}(4)$, which, once added to the indicated power, ensures the assessment of the mechanical power $\mathrm{P}_{\text {mech }}$ (measured on the pump shaft by a torque meter). The mechanical power was lost quite exclusively for the dry friction phenomena taking place between blade tip and stator inner surface (4) being equal to:

$$
P_{\text {mech,lost }}=f N_{\mathrm{V}} F_{\mathrm{N}} R_{\mathrm{a}} \omega
$$


This loss contribution is proportional to the number of vanes $\mathrm{N}_{\mathrm{V}}$, distance between blade tip and rotor center $R_{a}$ and the normal force $F_{N}$ exerted on the stator by the blades. It is important to observe that $\mathrm{F}_{\mathrm{N}}$ is given by the sum of the centrifugal force and the pressure force due to the fluid which fills the space under the blades (Fig. 1). Friction factor $\mathrm{f}$ was identified through the experimental validation; it was found equal to 0.2 which is in accordance to the typical values of materials of the components in relative motion. Once the mechanical power was known, the pump efficiency $\eta_{\text {pump }}$ can be evaluated according the radio between $\mathrm{P}_{\text {hydr }}$ and $\mathrm{P}_{\text {mech }}$ as reported in (5):

$$
\eta_{\text {pump }}=\frac{P_{h y d r}}{P_{\text {mech }}}
$$

Other friction losses (motion of the blade inside the slot) demonstrated to be negligible with respect to the contribution in (4). Finally, the model integrates a NIST ${ }^{\mathrm{TM}}$ real fluid properties database which allows to reproduce the real fluid behaviour mainly due to temperature.

\section{SVOP model experimental validation}

In order to use the numerical model of the SVOP as a software platform for machine design and as a tool to predict the machine performance when operating conditions change, the model was experimentally validated thanks to a wide campaign. The pump used as test case was a SVOP with a nominal displaced volume $V_{\text {disp }}$ of $74 \mathrm{~cm}^{3}$ which can be changed according the operating condition varying the pump eccentricity. Main relevant geometric variables are confidential and were not reported in this paper; on the other hand, the capability of the mathematical model to reproduce real behaviour is presented in the followings. In Fig. 2 , the experimental results (black point) referring to the nominal eccentricity case were reported in terms of Volumetric flow rate (Q)- Pressure Rise $(\Delta \mathrm{p})$ curves (a), absolute values of $P_{\text {mech }}(b), \eta_{v o l}(c)$ and $\eta_{\text {pump }}(\mathrm{d})$. The oil temperature at the intake is $115^{\circ} \mathrm{C}$. The results show as the machine its able to cover a huge range of operating condition when its revolution speed $\omega$ increases from $\omega_{1}$ to $\omega_{7}$. The $\mathrm{Q}-\Delta \mathrm{p}$ curves reported in Fig. 2(a) show this high flexibility in terms of Q (ranging from 40 to value exceeding $200 \mathrm{~L} / \mathrm{min}$ ) for a $\Delta$ p comprised between 1 to 9 bar. These curves assume the typical trend of a SVOP where the deviation from the theoretical Q was due to volumetric losses which increases when the pressure rise grows. In Fig. 2(b) $\mathrm{P}_{\text {mech }}$ was reported in correspondence of the operating point described by the Q- $\Delta \mathrm{p}$ curves. It can be observed as $\mathrm{P}_{\text {mech }}$ increases with mass flow rate keeping constant the $\Delta \mathrm{p}$, with absolute values comprised in a range between $100 \mathrm{~W}$ and $5000 \mathrm{~W}$. On the other hand, for a certain revolution speed, $\mathrm{P}_{\text {mech }}$ increases with a trend similar to $\mathrm{Q}-\Delta \mathrm{p}$ curves. Concerning $\eta_{\mathrm{vol}}$ (Fig. 2(c)), the SVOP allows to keep this performance parameter in a range comprised between 0.8 and 0.95 . Varying $\mathrm{Q}, \eta_{\mathrm{vol}}$ assumes a parabolic trend reaching the maximum values for Q comprised between 120 and $160 \mathrm{~L} / \mathrm{min}$. Finally, in Fig. 2 (d) the pump efficiency $\eta_{\text {pump }}$ values were reported. As it can be observed in Fig. 2(d) $\eta_{\text {pump }}$ diminishes with $Q$ reaching the maximum value (0.78-0.7) for $\mathrm{Q}$ comprised between $40-120 \mathrm{~L} / \mathrm{min}$. However, high $\eta_{\text {pump }}(0.8-0.6)$ can be kept for a wide operating range. The experimental results were compared with the numerical one (solid lines) evaluated through the model in correspondence of the same operating conditions. The results reported in Figure 3 show how the errors between experimental and numerical data can be considered quite satisfactory. Indeed, for Volumetric flow rate provided by the pump (Fig. 2(a)) the Root Mean Square Error (RMSE) is equal to $2 \%$ while the maximum deviation is $8.4 \%$ and takes place in correspondence of the highest $\omega$ probably due to blade instabilities which breaks the linearity of the last experimental Q- $\Delta$ p curves. For what concerns $P_{\text {mech }}$ (Fig. 2(b)) the results show that the model 
is able to reproduce the real mechanical power on the shaft as the RMSE is $7.1 \%$ and the maximum deviation $17 \%$ observed in correspondence of the highest $\omega\left(\omega_{7}\right)$.

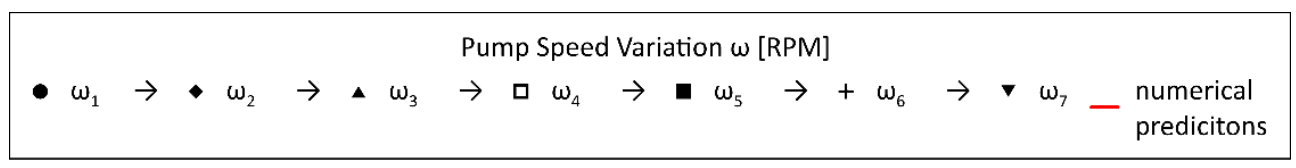

(a)

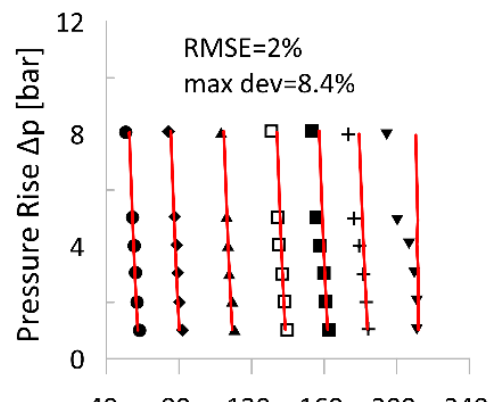

$\begin{array}{llllll}40 & 80 & 120 & 160 & 200 & 240\end{array}$

Volumetric flow rate $\mathrm{Q}[\mathrm{L} / \mathrm{min}]$

(c)

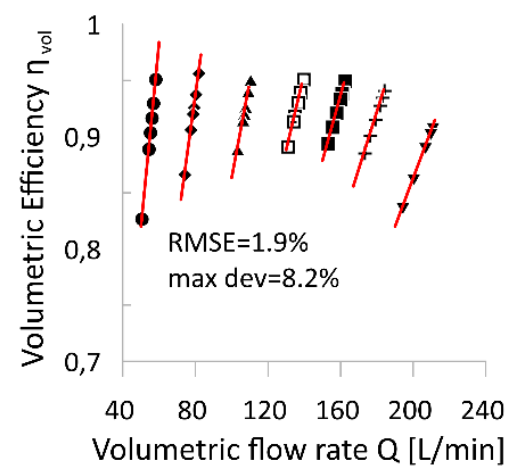

(b)

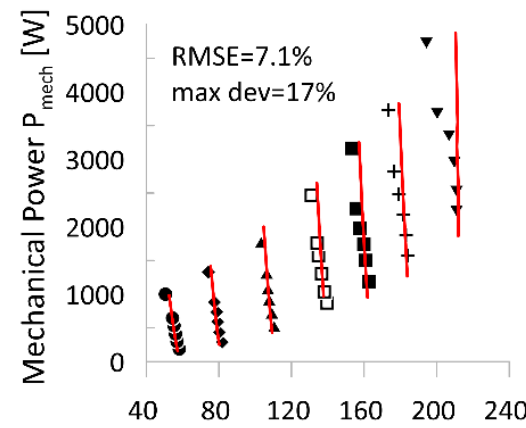

Volumetric flow rate $\mathrm{Q}[\mathrm{L} / \mathrm{min}]$

(d)

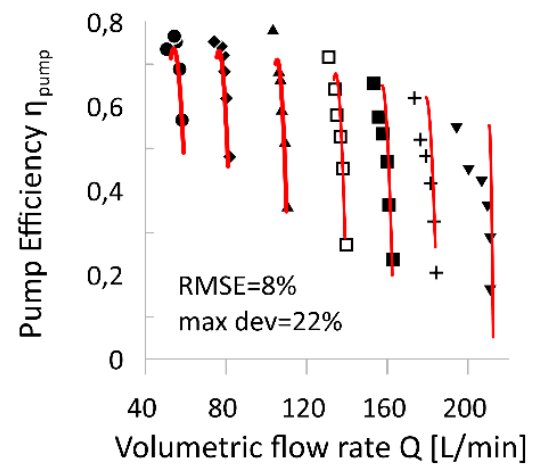

Fig. 2. Comparison between experimental and numerical data in terms of $Q(a)$, absolute values of $P_{\text {mech }}$ (b), $\eta_{\mathrm{vol}}(\mathrm{c}), \eta_{\text {pump }}$ (d) (nominal eccentricity) when $\omega$ increases from $\omega_{1}$ to $\omega_{7}$.

Also $\eta_{\mathrm{vol}}$ is well represented, but this is a consequence of the capability of the model to returns a correct representation of the experimental Q. Indeed, as it can be observed in Fig. 2(c) the RMSE and maximum deviation assumes values close to those of $\mathrm{Q}$ as they are respectively equal to $1.9 \%$ and $8.2 \%$. Concerning the $\eta_{\text {pump }}$, the model ensures a correct representation as it can be seen from Fig. 2(d) where the numerical predictions (solid lines) match with satisfying accuracy the experimental data. The goodness of representation is confirmed by the RMSE equals to $8 \%$ and a maximum deviation of $22 \%$. 


$$
\begin{array}{|l}
\text { Pump Speed Variation } \omega \text { [RPM] } \\
\text { - } \omega_{1} \rightarrow \quad \square \quad \omega_{2} \rightarrow \quad \omega_{3}-\text { numerical predictions }
\end{array}
$$

(a)

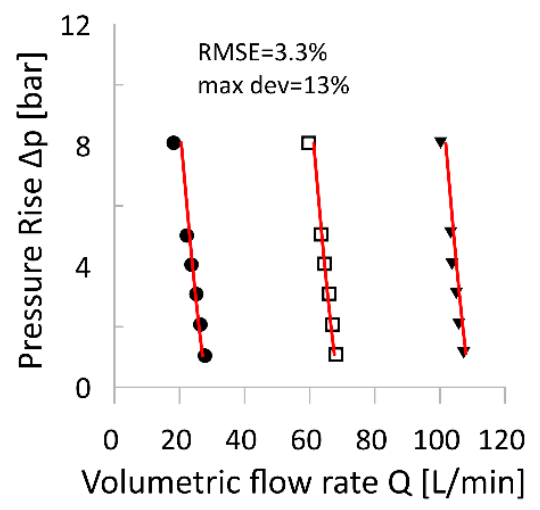

(c)

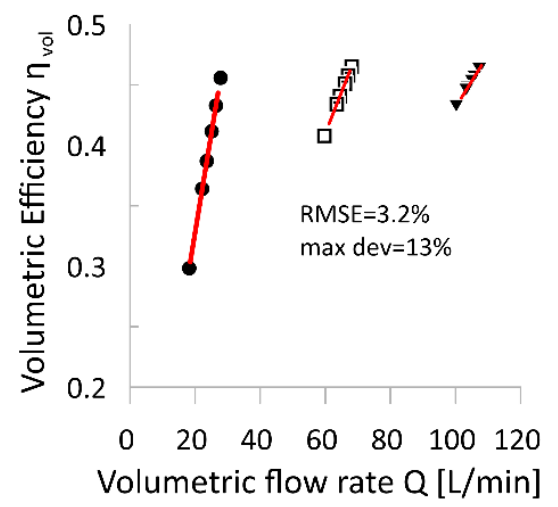

(b)

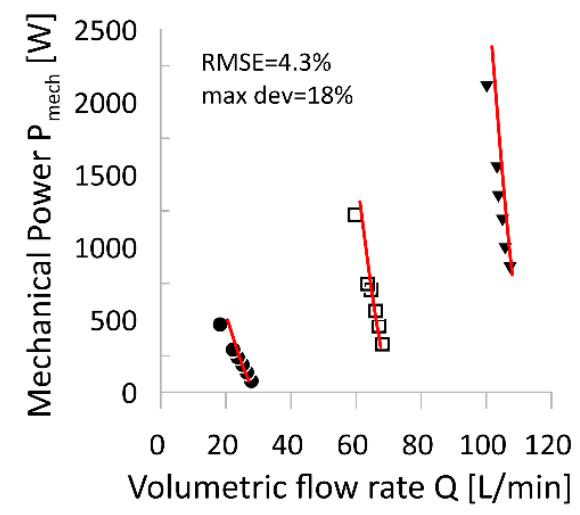

(d)

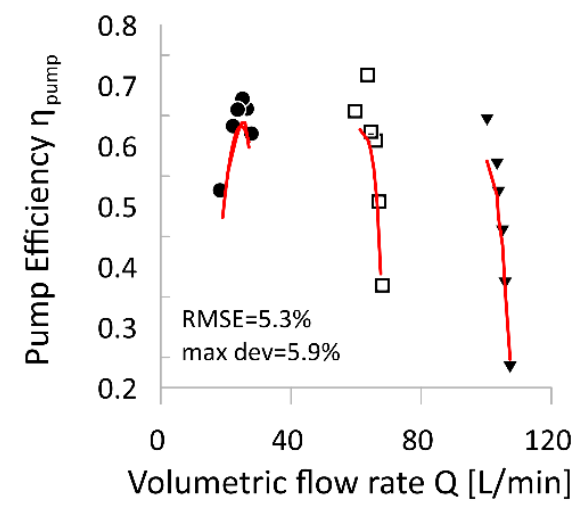

Fig. 3. Comparison between experimental and numerical data in terms of $Q(a), P_{\text {mech }}(b), \eta_{\text {vol }}(c)$, $\eta_{\text {pump }}(\mathrm{d})$ with an eccentricity reduction of $50 \%$ when $\omega$ increases from $\omega_{1}$ to $\omega_{3}$.

Fig. 2 considered a situation with maximum eccentricity. However, in order to evaluate the capability of the model to catch the SVOP performance also when the SVOP volume capacity varies (under regulation), the numerical results were compared to the experimental ones collected when the SVOP operates with the eccentricity reduced up to $50 \%$. The results of the comparison were reported in Fig. 3, and the good agreement between experimental and predicted data indicates that the model is able to represent the SVOP behaviour also when it works with a reduced volumetric capacity. Indeed, RMSEs of the errors on Q (Fig. 3(a)), absolute $\mathrm{P}_{\text {mech }}$ (Fig. 3(b)), $\eta_{\text {vol }}$ (Fig. 3(c)) and $\eta_{\text {pump }}$ (Fig. 3(d)) are respectively equals to 3.3 $\%, 4.3 \%, 13 \%$ and $5.3 \%$ while the maximum deviations are respectively $13 \%, 18 \%, 13 \%$ and $5.9 \%$. It is worth to observe that when the machine works with a reduction of its volume capacity, the volumetric efficiency and the pump efficiency decrease. This is due to the recirculation of part of the fluid at the sealing arc (tangency). Volumetric losses grow with eccentricity reduction and lead to a decrease of the same magnitude of volumetric efficiency. 
In fact, the volumetric efficiency of the SVOP operating with a $50 \%$ reduction of its maximum eccentricity (Fig. 3(c)) produces a similar reduction of nominal $\eta_{\text {vol. }}$. Moreover, thanks to the numerical model it was observed that when SVOP works with a $50 \%$ reduced capacity, the volumetric losses increases also at tip blade but the main contribution which affects $\eta_{\mathrm{vol}}$ is the flow recirculation at seal arc which leads also to a power loss of the machine. Indeed, the flow recirculated was compressed by the machine but it was not delivered. This aspect causes the reduction of pump efficiency (Fig. 3(d)).

\section{Analysis of regulation strategy on SVOP performance}

The experimental validation of the model ensures that it is able to reproduce the SVOP performance when its eccentricity (and consequently displaced volume $V_{\text {disp }}$ ) and revolution speed were varied for flow rate control. Thus, the model was used to compare these two control strategies: the variation of eccentricity $\left(\mathrm{V}_{\mathrm{disp}}\right)$ and that of the revolution speed. A wide range of situations were considered (a flow rate from 50 to $220 \mathrm{~L} / \mathrm{min}$ and a $\Delta \mathrm{p}$ from 1 to 8 bar). The model allows to evaluate an operating map for each initial operating point considered. In Figure 4 the map was reported for a SVOP rotating at a certain $\omega$ ( $\omega_{2}$ of Fig.3). Fig. 4(a) and Fig. 4(b) show, respectively, the effects of eccentricity reduction $\left(V_{\text {disp }}\right)$ on the flow rate delivered by the machine and on $\eta_{\text {pump. }}$. Similarly, the effects of the speed variation of the machine on the flow rate was reported (Fig. 4(c)) and on $\eta_{\text {pump }}$ (Fig. 4(d)) were assessed considering machine operating at rated eccentricity. The map easily allows to evaluate what is the best way to control the flow rate of the machine. In fact, in the case of flow rate control obtained modifying the eccentricity, entering in Figure $4 \mathrm{a})$ the current values of $\Delta \mathrm{p}$ (X-axis) and $\mathrm{Q}$ (as parameters of the lines in the figure), the $\mathrm{Y}$-axis gives the exact value of the eccentricity when the speed of the pump is $\omega_{2}$. On the contrary, for the same couple $(\Delta p, Q)$, Fig. 4(c) gives the pump speed if the eccentricity is at rated (maximum) value. Considering, for instance, that the new operating conditions are $\mathrm{Q}=60 \mathrm{~L} / \mathrm{min}$ and $\Delta \mathrm{p}=3$, fixing the pump speed at $\omega_{2}$, according to Fig. 4(a) the eccentricity should be set at $37 \%$ of the rated eccentricity; entering this eccentricity value in Fig. 4(b) (in correspondence with the same $\Delta \mathrm{p}$ ), it can be found $\eta_{\text {pump }}=0.35$. This efficiency decrease (with respect to the rated value) is due to losses for flow recirculation at seal arc. If the same flow rate $(60 \mathrm{~L} / \mathrm{min})$ and $\Delta \mathrm{p}(3$ bar) are obtained modifying the speed of the pump, keeping the eccentricity at maximum value, the speed must be set at $41 \%$ of $\omega_{2}$ - Fig. 4 (c) - and the pump efficiency will be close to 0.7 - Fig. 4(d) -, two times the value achieved with the eccentricity variation. Indeed, in this case there is no penalization of volumetric efficiency and consequently of the pump efficiency. More generally, varying the pump speed it is possible to cover a wide operating range keeping $\eta_{\text {pump }}$ close to the design values $(0.6-0.7)$. Thus, the operating maps in Figure 4 indicate that the best regulating strategy is that based on speed variation due to the absence of flow recirculation. However, this strategy needs to decouple the pump speed from the that of the engine. The best way is to make reference to an alternative actuation. Indeed, for flow rates $\mathrm{Q}$ slightly different from the rated value, the eccentricity reduction required is low and consequently the impact on $\eta_{\text {pump }}$ was limited. Indeed, observing Fig. 4(a) and Fig. 4(b) it can be noticed that for an absolute reduction of $Q$ equal to $20 \mathrm{~L} / \mathrm{min}$, a $18 \%$ eccentricity reduction from the rated case will be needed with a negligible pump efficiency decrease equal to 0.05 . This indicates that an eccentricity-based control does not involve significant detrimental effect on $\eta_{\text {pump }}$ if the operating points are not far from the rated ones. Therefore, the maps presented in Fig. 4 can be used not only for control purpose but also to define suitable values of the machine design. 
(a) Volume Flow Rate $Q$ [L/min]

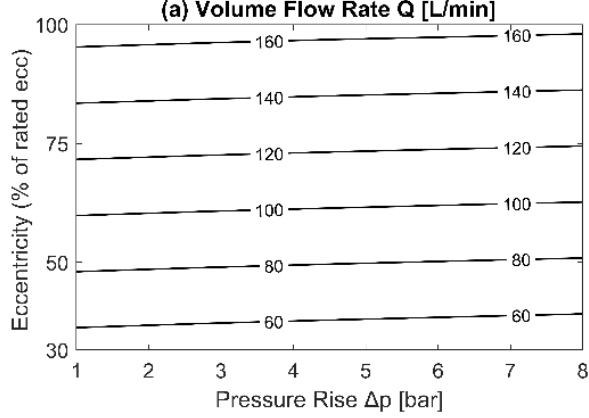

(c) Volume Flow Rate $Q$ [L/min]

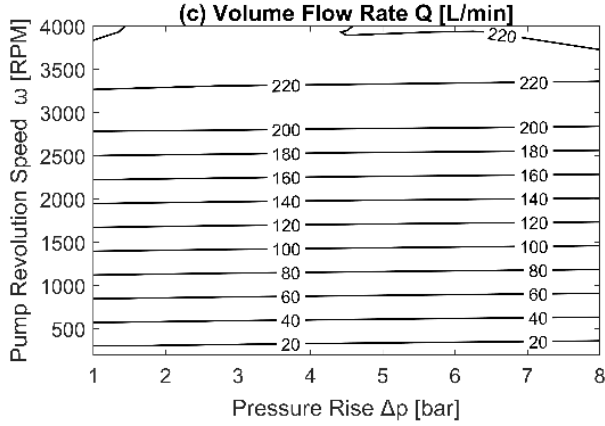

(b) Pump Efficiency $\eta_{\text {pump }}$

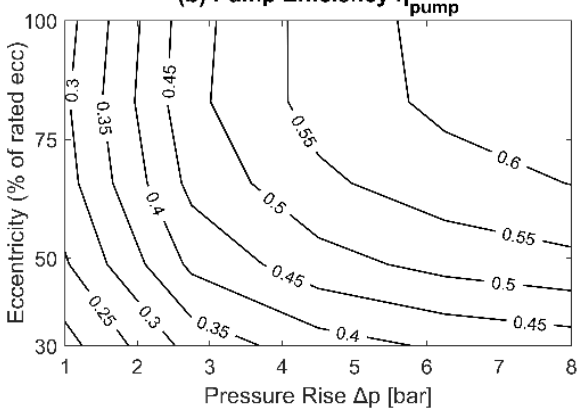

(d) Pump Efficiency $\eta_{\text {pump }}$

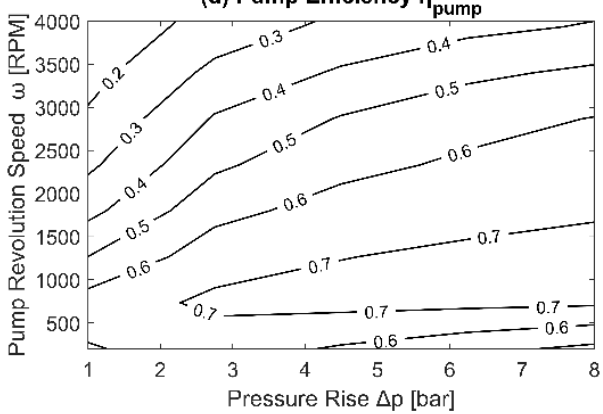

Fig. 4. Effect on Q provided by the pump (a) and pump efficiency (b) when SVOP eccentricity varies (a); Effect on Q provided by the pump (c) and pump efficiency (d) when SVOP eccentricity varies (a);

\section{Conclusions}

In the present paper a numerical model of a SVOP for the oil circulation in ICE was developed. The model was conceived following an integrated mono- and zero-dimensional approaches fully physically consistent and it was validated thanks to a wide experimental campaign conducted on an oil pump having $74 \mathrm{~cm}^{3}$ as design displacement. The agreement between theoretical and experimental data were very good for a wide set of variations of the pump speed and eccentricity (by reorienting the stator with respect to the rotor). Thanks to this agreement, two flow rate control were studied varying the pump speed or the eccentricity, for a given new operating point of the pump different from the rated one. The effect on pump efficiency of these two control strategies were summarized in a map which could be stored as look-up table. Looking at the pump efficiency, the comparison between the two control strategies shows:

The strategy based on the revolution speed variation (keeping constant the eccentricity at the rated value) demonstrates to be preferred. Indeed, when the maximum eccentricity is kept constant, the volumetric efficiency of the pump remains high, being the flow recirculation inside the machine prevented. Moreover, the speed variation requires the decoupling between the engine speed and pump speed. An alternative actuation is needed; 
- Despite the strategy based on eccentricity variation shows lower pump efficiency than the other one, it can keep a mechanical connection between pump and engine. The modification of the eccentricity can be obtained reorienting the stator with respect to the rotor. If the oil flow rate variations are limited, the pump efficiency decrease with respect to the rated value is reduced and the eccentricity variation can be accepted.

\section{Acknowledgements}

This work was developed during an intensive research program with O.M.P. Officine Mazzocco Pagnoni S.r.L. The authors are deeply grateful to the O.M.P. Officine Mazzocco Pagnoni S.r.L. for the possibilities to work together and to use experimental results to validate the numerical model.

\section{References}

1. E. G. Ribeiro, W. B. Melo, A. P. A. Filho, SAE Technical Papers, 1-7 (2005).

2. Yamamoto, K., Sadakata, N., Okada, H., \& Fujita, Y. (2017). SAE Technical Papers, 2017-March (March), 1-5. https://doi.org/10.4271/2017-01-1229

3. S. Loganathan, R. Sivanantham, J. Kumar, S. SAE Technical Papers (2009).

4. R. Sivanantham, J. Sureshkumar, SAE Technical Papers (2010).

5. S. Srinivasan, J. Suresh Kumar, K. Vijayakumar, P. Venkataraman, S. Loganathan, SAE Technical Papers (2012).

6. S. Loganathan, M. Anand, V. Vikraman, R. Vikas, SAE Technical Papers (2014)

7. A. Thakur, M. T. Alam, V. Kumar Ps, P. D. Kulkarni, S. Pandian, SAE Technical Papers, 5-8 (2017).

8. E. Frosina, A. Senatore, D. Buono, M. U. Manganelli, M. Olivetti, Energy Procedia, 45, 938-948 (2014).

9. M. Yamamoto, T. Hosogi, T. Watanabe, Y. Nishida, SAE International Journal of Fuels and Lubricants, 10 (3), 904-912 (2017).

10. J. Meira, A. Filho, W. Melo, E. Ribeiro, SAE Technical Papers (2011).

11. S. Loganathan, S. Govindarajan, J. Suresh Kumar, K. Vijayakumar, K. Srinivasan, SAE Technical Papers. (2011)

12. T. Arata, N. Novi, K. Ariga, A. Yamashita, G. Armenio, SAE Technical Papers (2012).

13. P. B. Kandavalli, R. Karthi, S. Suresh Kumar, M. Anand, (2017). SAE Technical Papers, 3-6 (2017).

14. J. Harrison, R. Aihara, M. Eshraghi, I. Dmitrieva, SAE Technical Papers, 1 (2014).

15. S. Jayanthamani, R. Sivanantham, M. Ibrahim, SAE Technical Papers (2019).

16. Rundo, M., \& Altare, G., Journal of Fluids Engineering, Transactions of the ASME, 140 (2018)

17. Rundo, M., Squarcini, R., \& Furno, F., SAE International Journal of Engines, 11(2), (2018)

18. Barbarelli, S., Bova, S., \& Piccione, R, SAE Technical Papers, (2009)

19. Gamma Technology. GT Suite ${ }^{\text {TM }}$ Flow Manual

20. F. Fatigati, M. Di Bartolomeo, R. Cipollone, Energy, 192, (2020). 